\title{
Relationship between Personality Types A and B and Creativity Level of Secondary School Students' In Kwara State, Nigeria
}

\author{
Bibire Abdulkareem Hussein \\ Department of Psychology Nigeria Police Academy Wudil, Kano State
}

\begin{abstract}
The study investigated the relationship between personality types and creativity level of secondary school adolescents' in Kwara State. Correlational research method was adopted. Stratified random sampling technique was used to select ten schools across the state. Four hundred students across the ten schools were sampled. Two instruments were used. Thus, Jugg-Myers Briggs Personality questionnaire and creativity and innovation scale adopted from Animasahun. The instruments were subjected to pilot testing and were found to have the coefficient of reliability of 0.76 and 0.68 respectively using the Pearson product moment correlation coefficient statistics at 0.05 level of significance. Findings of the study revealed that there was a significant relationship between personality types and creativity level of secondary school students in kwara state; it also revealed that there was no significant relationship between the variables on the basis of gender and school types. Recommendations were made by the researchers that attention should be given to students irrespective of their personality types by both parents and teachers in order to cater for them and provide necessary attention that can help them to explore and utilize their creative potentials, also teachers should use appropriate teaching methods in teaching in order to enhance students' creativity.
\end{abstract}

Keywords: Creativity, Personality Types, Relationship, students.

\section{Introduction}

Everyone is different, yet we also have several things in common. Coming to terms with human individuality and what makes an individual personality is one of the most fascinating questions in psychology. The model of personality which we hold is crucial for our perception of human beings It gives us our underlining beliefs about what human beings are really like (Hayes, 1994). Personality could be described using particular traits and types for example, the person's sociability, actions, impulse and speech pattern. Makin and Cooper (1981) opined that every day language is full of words to describe personality e g Shy, aggressive, dominant, ingratiating etc these patterns of behaviours the relatively stable can be used to predict the individual's future behaviour in similar or nearly all the situation.

In using these descriptions, we are attempting to generalize in a few words of a person's usual or preferred way of behaving, thinking and feeling which characterize an individual. Personality therefore, can be seen as the relatively enduring and stable patterns of behaving, thinking and feeling which characterize an individual. There are many different psychological theories of personality but they all have one thing incommon. They attempt to simplify the more important ways of describing individual patterns, of behaviour into a more manageable and measurable number of dimensions:

Psychology does not regard personality as a passive entity but as the dynamic character of the individual which finds expression through his conduct and activities. It's important to realize that no-one fully knows the extent to which personality is determined by genetics and hereditary factors, compared to the effects of up-bringing, culture, environment and experience. Nature versus Nurture: no-one knows. Most studies seem to indicate that it's a bit of each, roughly half and half, although obviously it varies person-to- person Given that perhaps half our personality is determined by influences acting upon us alike we are conceived and born, it's interesting and significant also that no-one actually knows the extent to which personality changes over time.

Primarily, it is important to be aware that personality characteristics play a significant role both in the act of learning and attitudes towards the act of learning. Variations in performance are not entirely a question of intellect, motivation or thinking skills, but may depend on the personal attributes which can enhance or inhibit the quality of that performance as well as attainment.

In order to emphasize the variety of personality pattern found in every classroom as the resultants of distinct biological background and varied experiences, the gambler, who ventures far beyond the limits of conservatism; the rebel, who reacts strongly against even the suggestion of domination; the, leader who understands people well enough to enjoy manipulating them; the narcissist, who is every fond of showing off for the sake of public applause. Such types are merely convenient labels for persons who carry to an extreme some very human characteristics. Indeed, at the moderate level most of these attitudes can contribute to the well - 
rounded personality. When self - centeredness is reduced to a consideration of the consequences to oneself, as well as to others, of a proposed social action, personality is likely to be strengthened by such concern.

Thus, the teacher who recognizes a common personality type in a learner can use an understanding of the characteristics involved in guiding development wisely, when used as a hit in designing a programme that leads towards wholesome balancing, the personality characterization serves usefully. Any typing, however, that assumes that these extreme characteristics are fixed, either by heredity, by subconscious' experiences of early infancy or the 'heavy hand of the culture's customs during childhood or later years, may become an obstacle to the personality adjustment and development, for which the school is partly responsible. The variety of personality types in every school class actually offers the teacher opportunities for arranging beneficial interpersonal contacts which in the group to promote the open ideal envisioned for each differing learner.

Friedman, and Rosenman (1974) have identified a pattern of personality characteristics which they referred to as Type A behaviour pattern and which they claim is associated with the development of heart disease This pattern includes what Friedman and Rosenman call hurry sickness a habitual sense of time urgency Type A behaviour pattern as defined by Friedman and Rosenman (1974), is an action emotion complex that can be observed in any person who is aggressively involved less and less time and if required to do so against the efforts of others or things or persons in the same environment. The major behavioural manifestations of a Type A person as revealed by Friedman and Rosenman, include extreme competitive achievement striving, chronic sense of time urgency, impulsivity and extreme aggressive tendency which are often characterized by free floating hostility that could be aroused by challenges.

In contrast to Type A persons are Type B persons who may be just as ambitious and intelligent as the type A person but who do things in a slower, more relaxed and Secured way. For the fore going emphasizes, it is evident, that people can be classified into categories based on their personality characteristics. Personality type developed by Jung in the early 1920 had of recent resurrected and made into a practical instrument for measuring behaviour through Myers-Brigg's Type indicator (MBTI) (Nori.ng 1993). This personality type is most widely used in career counseling and development and education. Therefore personality can be grouped under two major personality types, namely personality type A and B personality type. The categorization refers to distinct and discontinues types such as you are either one or the other.

The following are the characteristics of the Type A personality

1. Type A's have got a severe sense of time urgency. They are also always running and can hardly relax, if they sat without doing something useful they may end up feeling guilty.

2. Type A's are over achievers, they usually get themselves involved in many different unrelated activities and perform well in them all

3. Type A's biggest problem is stress; they are usually overwhelmed by the amount of tasks they have to do. These tasks are usually a huge list that they planned for themselves. They type A person is usually competitive and has a high challenging spirit.

4. A Type A personality is prone to angry outbursts. The cause of this hostility towards people is usually due to them not meeting the high expectation of the type A personality. Anger management is an important element in modifying this form of aggression and develop self control.

5. Winning is all important to the type A personality. They will compete in all effects, no matter who it is against and what is the prize.

6. A type A's are adapt at multitasking, but tend to be very impatient and are irritated by delays and people they consider incompetent. Everything is a rush to the Type A so they will demand people to hurry up. Type A's are unable to relax and take time out to sit quietly doing nothing. Take time to reflect and slow down your pace. Jenksin, Ayzanski \& Roseman (1971).

In contrast, type B individuals are calm and have an easy-going attitude. They are fun-loving and are relatively less competitive. They are also better at relaxing without feeling guilty and they work without getting anxious or agitated. Type B's are the opposite of Type A's. They are laid back and not easily stressed. While type B can be achievers too, still they won't be as competitive as Type As'. Type B's can delay work and do it in the last ,moment, of them can turn into procrastinators which is something that Type A can never do Alao (1989).

Creativity is a special kind of thinking that involves originality and fluency, which breaks away from existing patterns and introduces something new. Creativity may be applied to problem solving, in which ease it facilitates the generation of range of possible solution, in particular to problems which have no single right answer. Alternatively, and most productively, it may be applied to the process of creating. This means the realizing of a held vision, empowered by a tension-resolution system which is put in place by the existing reality being differentiated from a desired vision. Creativity is obviously something that happens frequently in everyday life, rather than something confined to poets, painters and musicians. The conceptual meaning of 
creativity can be supplemented with an experiential definition; it is believed that creative individual can be generally described as a fellow who has a higher score in open-ended test than in intelligence tests.

Akinboye (1997) asserted that creativity is a characteristic that a person possesses to produce a product that is regarded as original and a process by which an unusual thing is obtained. Personality characteristic tend to table associated with a medium of expression or any other features of creative production For example, the outstanding artist looks more like less competent artist while the great sculptor. Therefore, both profession and public view acknowledgement that the maker of creative product looks different from his fellow men such difference could be cognitive, personality way of life or stylistic quality of the product. There are, however, variations in creativity in spite of the fact that creativity follows a predictable pattern. One of the factors that are responsible for these variations is gender. Ortese (2003) pointed out that boys show greater creativity than girls, especially as childhood advances. In a larger part, this is due to the different treatment boys and girls receive. While boys are given more opportunities to be independent, they are pushed by peers to take more risks. They are also encouraged by parents and teachers to show more initiative and originality. Girls on their part are confined to the home and encouraged to be tender, gentle and careful. According to Tunde (2008) private school students attained high level of creativity than their counterparts in public school regardless of their personality types. Bibre (2010) submitted that students with personality type A attributes have high level of creativity and academic attainment than students with personality type B characteristics or attribute.

\section{Purpose of Study}

The main purpose of this study is to find out the relationship between personality types and creativity level of Secondary Adolescents' in Kwara State, Nigeria. Specifically this study aimed at finding out the:

1.1 Relationship between personality types A and B and creativity level of secondary school adolescents in Kwara State on the basis of gender.

1.2 Relationship between personality types A and B and creativity level of adolescents in private and public schools in Kwara State

\section{Research Questions}

The following research questions were generated to sharpen the focus of the study.

1. What is the relationship between personality types A and B and creativity level of secondary schools adolescents in Kwara State?

2. What is the relationship between personality types A and B and creativity level of secondary school adolescents on the basis of gender?

3. What is the relationship between personality types A and B and creativity level of secondary school adolescent in public and private schools in Kwara State.

\section{Hypotheses}

The following null hypotheses were derived for testing in this study.

$\mathrm{Ho}_{1}$ : There is no significance relationship between the personality types A and B and creativity level of secondary schools adolescents in Kwara State.

$\mathrm{Ho}_{2}$ : There is no significance relationship between the personality types A and B and creativity level of male and female secondary school adolescents in kwara state.

$\mathrm{Ho}_{3}$ : There is no significance relationship between the personality types A and B and creativity level of secondary school adolescents on the basis of school type.

\section{Methodology}

The study is correlational survey type. The method was considered appropriate for this study since the researcher is interested in finding out the relationship between personality types and creativity level of secondary school adolescents in Kwara Sate. All senior secondary school students in Kwara State constitute the study population. The target population for the study was students selected randomly selected secondary schools in the state, in all four hundred (400) students, randomly selected from the ten (10) schools constituted the sample.

The researcher used stratified random sampling technique to select four secondary schools from Kwara South. Two public and private schools were selected from Kwara central, two public and one private school were selected from Kwara South and two public and one private school were selected from Kwara North. Simple random sampling Technique was used to select forty (40) senior secondary schools students in each of the ten schools in each senatorial district, for a total of four hundred respondents. Two hundred and fifty (250) male respondents were selected while one hundred and fifty (150) respondents were also selected across the ten schools, making a total number of our hundred (400) respondents. 


\section{Instrumentation}

The research instruments used for the study were the questionnaires, adopted from personality test based on Jung-Myers- Brigg typology (1998) and it was used to measure the personality types of each student. The questionnaire was divided into two sections. Section A elicited the demographic characteristics of the respondents while section B contained forty (40) items to elicit responses which were used to categorized respondents into personality types. Students were required to tick either "yes" or "No" in response to each item.

The second instrument used was creativity and innovation scale (CIS) adopted from Animasahun (2007). The test contains thirty-three items.

To establish the validity of the instruments the researcher gave copies to experts for face and content validity. To establish the reliability of the instruments test re-test method was adopted.

\section{Procedure}

Consequent upon the granting of permission for administration of the instruments by the authorities of the selected secondary schools, the instruments were administered with the help of research assistants. The instruments were administered to the students and lasted for 1 hour after which there was immediate retrieval

\section{Data Analysis}

Frequency and percentage were used to answer demographic characteristics of the respondents while the Pearson product moment correction co-efficient statistic was employed to test the hypotheses.

\section{Presentation and Discussion of Findings}

The results obtained from the analysis of the research and the null hypotheses formulated in this study are show in tables below:

Research question 1: What is the relationship between personality types and creativity level of secondary schools students in Kwara State?

In order to answer this research question responses of the respondents on the questionnaire were categorized into high; average and low as shown in table.

\begin{tabular}{|l|l|l|l|}
\hline Creativity Levels & Personality Type A & Personality Type B & Total \\
\hline High: & $\mathbf{7 0}(52.2 \%)$ & $\mathbf{6 4}(\mathbf{4 7 . 8 \%})$ & $\mathbf{1 3 4 ( 1 0 0 . 0 \% )}$ \\
\hline Average & $\mathbf{7 1}(53.4 \%)$ & $\mathbf{6 2}(\mathbf{4 6 . 6 \%})$ & $\mathbf{1 3 3}(\mathbf{1 0 0 . 0 \%})$ \\
\hline Low & $\mathbf{5 3}(39.8 \%)$ & $\mathbf{8 0}(60.2 \%)$ & $\mathbf{1 3 3}(\mathbf{1 0 0 . 0} \%)$ \\
\hline Total & 194 & $\mathbf{2 0 6}$ & $\mathbf{4 0 0}$ \\
\hline & $\mathbf{4 8 . 5 \%}$ & $\mathbf{5 1 . 5 \%}$ & $\mathbf{1 0 0 . 0 \%}$ \\
\hline
\end{tabular}

TABLE 1 shows that 400 respondents participated in the study out which seventy $70(52.2 \%)$ personality type A respondents have high level of creativity seventy one $71(53.4 \%)$ have average of creativity and fifty three $53(39.80 \%)$ have low level of creativity. It could also be seen that sixty-four $64(42.8 \%)$ of personality type B respondents have high level of creativity, sixty two (46.6\%) have average level of creativity while eighty $80(60.2 \%)$ have low level of creativity.

Research question 2 and 3 were answered by corresponding hypotheses 2 and 3 respectively.

\section{Hypotheses Testing}

All the hypotheses formulated were tested with the use of a Pearson product moment correlation coefficient (PPMC) through the use of point biserial correlation that describes the association in a contingency table as shown below at alp ha level of 0.05 .

$\mathrm{HO}_{1}$ : $\quad$ There is no significant relationship between the personality types and creativity level of secondary schools students in Kwara State.

In order to test this hypothesis, response of the students to the questionnaire were collated and subjected to statistical analysis as shown

below.

TABLE 2: Pearson Personality Types in Kwara State $\mathbf{r}$ Analysis Showing the Relationship Between the Personality Types and Creativity Level of Secondary School Students

\begin{tabular}{|c|c|c|c|c|c|c|c|c|c|}
\hline Creativity levels & $\begin{array}{l}\text { Pers } \\
\text { A }\end{array}$ & Type & $\begin{array}{l}\text { Pers } \\
\text { B }\end{array}$ & Type & Total & Df & Cal-r Value & $\begin{array}{l}\text { Crit-r } \\
\text { Value }\end{array}$ & Remark \\
\hline High & 70 & & 64 & & 134 & & & & \\
\hline Average & 71 & & 62 & & 133 & 398 & .101 & 0.088 & Rejected \\
\hline
\end{tabular}

$\begin{array}{rrr}194 & 206 & 400\end{array}$

Significance at 0.05 alpha levels. 
TABLE 2 shows that the calculated " $r$ " value is .101 while the critical " $r$ " value is 0.088 with 398 degree of freedom and at alpha level of 0.05 . Since the calculated " $r$ " value is greater than the critical. "r" value hypothesis I was rejected and the alterative hypothesis was upheld that, there is a significant relationship between the personality types and creativity ability of secondary school students in Kwara State.

$\mathrm{H}_{02} \quad$ There is no significant relationship between personality types and creativity level of secondary school students on the basis of gender.

In order to test this hypothesis, responses of the students to the

Questionnaire was collated on the basis of gender and subjected to statistical analysis as shown below.

\begin{tabular}{|c|c|c|c|c|c|c|c|c|c|}
\hline & \multicolumn{5}{|c|}{ Male } & \multicolumn{4}{|c|}{ Female } \\
\hline Creativity levels & $\begin{array}{l}\text { Pers } \\
\text { Type A }\end{array}$ & $\begin{array}{l}\text { Pers } \\
\text { Type B }\end{array}$ & $\begin{array}{l}\text { Pers } \\
\text { Type } \\
\text { A }\end{array}$ & $\begin{array}{l}\text { Pers } \\
\text { Type } \\
\text { B } \\
\end{array}$ & Total & Df & $\begin{array}{l}\text { Cal-r } \\
\text { Value }\end{array}$ & $\begin{array}{l}\text { Crit-r } \\
\text { Value }\end{array}$ & Remark \\
\hline High & 33 & 50 & 33 & 16 & 132 & & & & \\
\hline Average & 27 & 57 & 30 & 20 & 134 & 398 & .060 & 0.088 & Accepted \\
\hline Low & 53 & 32 & 35 & 15 & 134 & & & & \\
\hline Total & 112 & 139 & 98 & 51 & 400 & & & & \\
\hline
\end{tabular}

Significance at 0.05 alpha levels.

TABLE 3 shows that the calculated ' $r$ ' value is .0 .60 while the critical ' $r$ ' values 0.088 with 398 degree of freedom and at alpha level of 0.05 . Since the calculated ' $r$ ' value is less than critical ' $r$ ' value, hypothesis 2 was accepted, that there is no significant relationship between the personality types and creativity level of secondary school students on the basis of, gender.

$\mathrm{H}_{03}$ : there is no significant relationship between the personality and creativity level of students in public and private schools in Kwara State.

In order to test this hypothesis, responses of the respondents to the questionnaire were collated on the basis of school types and subjected to statistical analysis as show below:

Public schools and private schools

\begin{tabular}{|c|c|c|c|c|c|c|c|c|c|}
\hline Creativity levels & $\begin{array}{l}\text { Pers } \\
\text { Type A }\end{array}$ & $\begin{array}{l}\text { Pers } \\
\text { Type B }\end{array}$ & $\begin{array}{l}\text { Pers } \\
\text { Type } \\
\text { A }\end{array}$ & $\begin{array}{l}\text { Pers } \\
\text { Type } \\
\text { B }\end{array}$ & Total & Df & $\begin{array}{l}\text { Cal-r } \\
\text { Value }\end{array}$ & $\begin{array}{l}\text { Crit-r } \\
\text { Value }\end{array}$ & Remark \\
\hline High & 60 & 20 & 23 & 30 & 133 & & & & \\
\hline Average & $\mathbf{5 0}$ & 30 & 28 & 25 & 133 & 398 & 0.45 & 0.088 & Accepted \\
\hline Low & 67 & 14 & 33 & 20 & 134 & & & & \\
\hline Total & 177 & 64 & 84 & 75 & 400 & & & & \\
\hline
\end{tabular}

Significance at 0.05 alpha levels.

TABLE 4 shows that the calculated ' $r$ ' value is 0.045 while the critical ' $r$ ' value is 0.088 with 398 degree of freedom and at alpha level of 0.05 . Since the calculated ' $r$ ' value is less than the critical ' $r$ ' value hypothesis 4 was accepted that there is no significant relationship between the personality types and creativity level of students in public and private schools in Kwara State.

\section{Discussion}

The findings of the study revealed that a significant relationship existed between personality types of students and their creativity level. This indicates that personality type A students have high level of creativity than their counterparts i.e personality type B. This finding corroborated with the finding of Bibire (2010) where he posited that personality type A students have high intelligence than personality type B individual because they performed academically better than their counterparts in their academic tasks. It can also be observed from the findings of this study that there was no significant relationship between the personality types and students creativity level on the basis of gender and school type. Findings of this study corroborated that: of Tunde (2008) where he asserted in his study that private school students attained high level of creativity than their counterparts in public school regardless of' their personality types. The result of this finding was in contrary to researches conducted by ortese (2003) pointed out that boys show greater creativity than girls, especially as child hood advances, in a large part, this is due to different treatment boys and girls received while boys are given more opportunities to be independent, they are pushed by peers to take more risks. They are also encouraged by parents and teachers to show more initiative and originality. Girls on their part are confined to the home and encouraged to be tender, gentle and careful. 


\section{Conclusion}

The following conclusions were drawn from the study

1. Significant relationship exists between personality types A and B of students and their creative level. This shown that personality type A students have high level of creativity than personality type B individuals.

2. Significant relationship does not exist between personality type A and B and students creativity level on the basis of gender and school type.

\section{Recommendations}

In view of the findings of this study the following recommendations were made

1. Attention should be given to the students irrespective of their personality types by parents and teachers so as to cater for them and provide necessary facilities that can help them to explore and utilize their creative potentials.

2. Teachers should also use appropriate methods in the teaching of their students in order to enhance their creativity

3. Teachers should allow students to use their divergent thinking, which promotes creativity, instead of convergent think which only promote rote learning.

\section{References}

[1] N. Hayes, Foundations of psychology: An introductory text, Harmondaworth: Penguin publisher. 1994

[2] P. Makin, \& C.L. Cooper, Psychology for managers. New York free press. 1981

[3] M. Friedman, \& R H Rosenman, Type A Behaviour and Your Heart, New York 1974

[4] J. Nuring, Almost the FAQ personality type summary. www. Nuring @.netcom. Com August 23, 1993.

[5] Jenksin, Ayzanski \& Roseman. A survey of personality Type A and B. Hosted by San Diego figure skating communications. 1971

[6] A, I. Alao Behaviour pattern and styles of achievement striving of Nigeria secondary school students. An unpublished doctoral thesis, department of guidance and counseling, university of Ilorin, Ilorin, Nigeria. 1989

[7] J.O. Akinboye,. Effect of synectics and brain storming strategies in fostering creativity in Nigerian adolescents, Sample Research Methods. Ibadan: University of Ibadan Press. 1997

[8] P.T. Ortese, Total development of man, the imperative of creativity. Being a paper presented at the 27 Annual National Conference of Co Association of Nigeria held at the University of Ibadan, Oyo State. I 8-22th August, 2003

[9] S.U. Tunde, Assessment of creativity of secondary school students in Ilorin, Nigeria. An M.ed Project Department of Arts and Social Sciences Faculty of Education, University of Ilorin, llorin Nigeria. 2009

[10] A.K.H. Bibre, Relationship between personality types and secondary school students academic achievement in Kwara State, Nigeria. An unpublished project M.Ed project submitted to the Department of Social Sciences Education University of Ilorin, Ilorin -Nigeria. 2010. 